\title{
SCHRODINGER'S CAT PARADOX RESOLUTION USING GRW COLLAPSE MODEL
}

\author{
J.Foukzon ${ }^{1}$, A.A.Potapov ${ }^{2}$, S.A.Podosenov ${ }^{3}$ \\ ${ }^{1}$ Israel Institute of Technology, ${ }^{2}$ IRE RAS, ${ }^{3}$ All-Russian Scientific-Research Institute
}

\begin{abstract}
:
Possible solution of the Schrödinger's cat paradox is considered.We pointed out that: the collapsed state of the cat always shows definite and predictable measurement outcomes even if Schrödinger's cat consists of a superposition: $\mid$ cat $\rangle=c_{1} \mid$ live cat $\rangle+c_{2} \mid$ death cat $\rangle$
\end{abstract}

\section{Keywords}

Measurement problem, two-state systems, GRW collapse model, stochastic nonlinear Schrödinger equation, Schrödinger's cat paradox.

\section{Introduction}

As Weinberg recently reminded us [1], the measurement problem remains a fundamental conundrum. During measurement the state vector of the microscopic system collapses in a probabilistic way to one of a number of classical states, in a way that is unexplained, and cannot be described by the time-dependent Schrödinger equation [1]-[5].To review the essentials, it is sufficient to consider two-state systems. Suppose a nucleus n whose Hilbert space is spanned by orthonormal states

$\left|\mathrm{s}_{\mathrm{i}}(\mathrm{t})\right\rangle, \mathrm{i}=1,2 \quad$ where $\quad\left|\mathrm{s}_{1}(\mathrm{t})\right\rangle=\mid$ undecayed nucleus at instant $\left.\mathrm{t}\right\rangle$ and $\left|\mathrm{s}_{2}(\mathrm{t})\right\rangle=$ |decayed nucleus at instant $t\rangle$ is in the superposition state,

$\left|\Psi_{\mathrm{t}}\right\rangle_{\mathrm{n}}=\mathrm{c}_{1}\left|\mathrm{~s}_{1}(\mathrm{t})\right\rangle+\mathrm{c}_{2}\left|\mathrm{~s}_{2}(\mathrm{t})\right\rangle,\left|\mathrm{c}_{1}\right|^{2}+\left|\mathrm{c}_{2}\right|^{2}=1$

An measurement apparatus A, which may be microscopic or macroscopic, is designed to distinguish between states $\left|\mathrm{s}_{\mathrm{i}}(\mathrm{t})\right\rangle, \mathrm{i}=1,2$ by transitioning at each instant $\mathrm{t}$ intostate $\left|\mathrm{a}_{\mathrm{i}}(\mathrm{t})\right\rangle, \mathrm{i}=$ 1,2 if it finds $n$ is in $\left|s_{i}(t)\right\rangle, i=1,2$. Assume the detector is reliable, implying the $\left|a_{1}(t)\right\rangle$ and $\left|\mathrm{a}_{2}(\mathrm{t})\right\rangle$ are orthonormal at each instant t-i.e., $\left\langle\mathrm{a}_{1}(\mathrm{t}) \mid \mathrm{a}_{2}(\mathrm{t})\right\rangle=0$ and that the measurement interaction does not disturb states $\left|s_{i}(t)\right\rangle, i=1,2$-i.e., the measurement is "ideal". When A measures $\left|\Psi_{t}\right\rangle_{\mathrm{n}}$, the Schrödinger equation's unitary time evolution then leads to the "measurement state" (MS) $\left|\Psi_{\mathrm{t}}\right\rangle_{\mathrm{nA}}$ : 
$\left|\Psi_{\mathrm{t}}\right\rangle_{\mathrm{nA}}=\mathrm{c}_{1}\left|\mathrm{~s}_{1}(\mathrm{t})\right\rangle\left|\mathrm{a}_{1}(\mathrm{t})\right\rangle+\mathrm{c}_{2}\left|\mathrm{~s}_{2}(\mathrm{t})\right\rangle\left|\mathrm{a}_{1}(\mathrm{t})\right\rangle,\left|\mathrm{c}_{1}\right|^{2}+\left|\mathrm{c}_{2}\right|^{2}=1 .(1.2)$

of the composite system nAfollowing the measurement. Standard formalism of continuous quantum measurements [2],[3],[4],[5] leads to a definite but unpredictable measurement outcome, either $\left|\mathrm{a}_{1}(\mathrm{t})\right\rangle$ or $\left|\mathrm{a}_{2}(\mathrm{t})\right\rangle$ and that $\left|\Psi_{\mathrm{t}}\right\rangle_{\mathrm{n}}$ suddenly "collapses" at instant $\mathrm{t}$ ' into the corresponding state $\left|s_{i}(t)\right\rangle, i=1,2$. But unfortunately equation (1.2) does not appear to resemble such a collapsed state at instant $\mathrm{t}^{\prime}$ ?. The measurement problem is as follows [7]:

(I) How do we reconcile canonical collapse models postulate's

(II) How do we reconcile the measurement postulate's definite outcomes with the "measurement state"

$\left|\Psi_{\mathrm{t}}\right\rangle_{\mathrm{nA}}$ at each instant $\mathrm{t}$ and

(III) how does the outcome become irreversibly recorded in light of the Schrödinger equation's unitary and, hence, reversible evolution?

This paper deals with only the special case of the measurement problem, knownas Schrödinger's cat paradox. For a good and complete explanation of this paradoxsee Leggett [6] and Hobson [7].

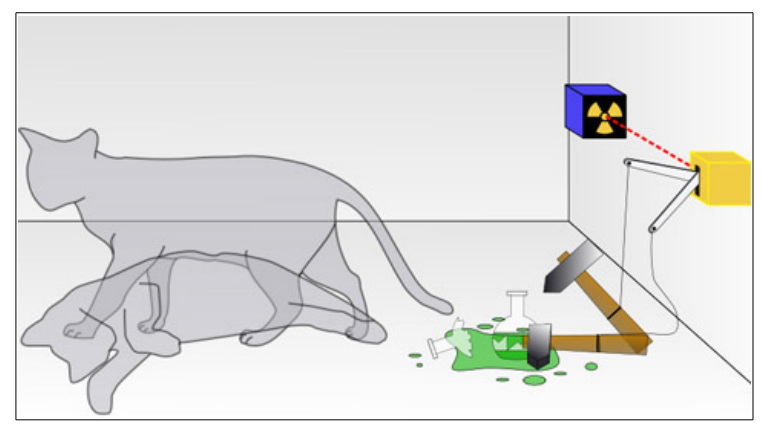

Pic.1.1.Schrödinger's cat.

Schrödinger's cat: a cat, a flask of poison, and a radioactive source are placed in a sealed box. If an internal monitor detects radioactivity (i.e. a single atom decaying), the flask is shattered, releasing the poison that kills the cat. The Copenhagen interpretation of quantum mechanics implies that after a while, the cat is simultaneously alive and dead. Yet, when one looks in the box, one sees the cat either alive or dead, not both alive and dead. This poses the question of when exactly quantum superposition ends and reality collapses into one possibility or the other.

This paper presents antheoretical approach of the MS that resolves the problem of definite outcomes for the Schrödinger's "cat". It shows that the MS actually is the collapsed state of both Schrödinger's "cat" and nucleus, even though it evolved purely unitarily.

The canonical collapse models In order to appreciate how canonical collapse models work, and what they are able to achieve, we briefly review the GRW model. Let us consider a system of $n$ particles which, only for the sake of simplicity, we take to be scalar and spin-less; the GRW model is defined by the following postulates [2] :

(1) The state of the system is represented by a wave function $\left|\psi_{t}\left(x_{1}, \ldots, x_{n}\right)\right\rangle$ belonging to the 
Hilbert space $L_{2}\left(\mathbb{R}^{3 n}\right)$.

(2) At random times, the wave function experiences a sudden jump of the form:

$$
\begin{gathered}
\psi_{\mathrm{t}}\left(\mathrm{x}_{1}, \ldots, \mathrm{x}_{\mathrm{n}}\right) \rightarrow \psi_{\mathrm{t}}\left(\mathrm{x}_{1}, \ldots, \mathrm{x}_{\mathrm{n}} ; \tilde{\mathrm{x}}_{\mathrm{m}}\right), \mathrm{m} \leq \mathrm{n}, \\
\psi_{\mathrm{t}}\left(\mathrm{x}_{1}, \ldots, \mathrm{x}_{\mathrm{n}} ; \tilde{\mathrm{x}}_{\mathrm{m}}\right)=\mathcal{R}_{\mathrm{m}}\left(\tilde{\mathrm{x}}_{\mathrm{m}}\right) \psi_{\mathrm{t}}\left(\mathrm{x}_{1}, \ldots, \mathrm{x}_{\mathrm{n}}\right)\left[\left\|\mathcal{R}_{\mathrm{m}}\left(\tilde{\mathrm{x}}_{\mathrm{m}}\right) \psi_{\mathrm{t}}\left(\mathrm{x}_{1}, \ldots, \mathrm{x}_{\mathrm{n}}\right)\right\|_{2}\right]^{-1}
\end{gathered}
$$

Here $\psi_{\mathrm{t}}\left(\mathrm{x}_{1}, \ldots, \mathrm{x}_{\mathrm{n}}\right)$ is the state vector of the whole system at time $\mathrm{t}$, immediately prior to the jump process and $\mathcal{R}_{\mathrm{m}}\left(\widetilde{\mathrm{x}}_{\mathrm{m}}\right)$ is a linear operator which is conventionally chosen equal to:

$$
\mathcal{R}_{\mathrm{m}}(\tilde{\mathrm{x}})=\left(\pi \mathrm{r}_{\mathrm{c}}^{2}\right)^{-3 / 4} \exp \left(\hat{\mathrm{x}}^{2} / 2 \mathrm{r}_{\mathrm{c}}^{2}\right),(1.4)
$$

wherer ${ }_{c}$ is a new parameter of the model which sets the width of the localization process, and $\hat{x}_{m}$ is the position operator associated to the $\mathrm{m}$-th particle of the system and the random variable $\tilde{\mathrm{x}}_{\mathrm{m}}$ which corresponds to the place where the jump occurs.

(3) It is assumed that the jumps are distributed in time like a Poisson process with frequency $\lambda=\lambda_{\mathrm{GRW}}$ this is the second new parameter of the model.

(4) Between two consecutive jumps, the state vector evolves according to the standard Schrödinger equation.We note that GRW collapse model follows from the more general S. Weinberg formalism [1].Another modern approach to stochastic reduction is to describe it using a stochastic nonlinear Schrödinger equation [2],[3],[4],[5].

\section{Generalized Gamow theory of the alpha decay via tunneling using GRW collapse model.}

By 1928, George Gamow had solved the theory of the alpha decay via tunneling [8]. The alpha particle is trapped in a potential well by the nucleus. Classically, it is forbidden to escape, but according to the (then) newly discovered principles of quantum mechanics, it has a tiny (but non-zero) probability of "tunneling" through the barrier and appearing on the other side to escape the nucleus. Gamow solved a modelpotential for the nucleus and derived, from first principles, a relationship between thehalf-life of the decay, and the energy of the emission. The $\alpha$-particle has total energy Eand is incident on the barrier from the right to left, see Pic.2.1. 


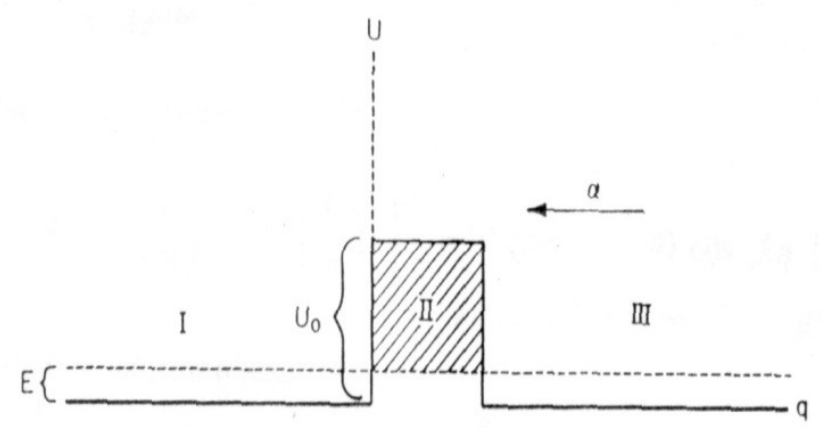

Pic. 2.1.The particle has total energy $E$ andis incident on the barrier $U(x)$ from right to left.

The Schrödinger equation in each of regions: $\mathbf{I}=\{x \mid x<0\}, \mathbf{I I}=\{x \mid 0 \leq x \leq l\}$ and $\quad$ III $=$ $\{x \mid x \geq 0\}$ takes the following form:

$\partial^{2} \Psi(x) / \partial x^{2}+2 m / \hbar^{2}[E-U(x)] \Psi(x)=0$.

Here (i) $U(x)=0$ in region I,(ii) $U(x)=U_{0}$ in region II, (iii) $U(x)=0$ in region III. The corresponding solutions reads [8]:

$\Psi_{\mathrm{I}}(x)=A \cos (k x), \Psi_{\mathrm{II}}(x)=B_{+} \exp \left(k^{\prime} x\right)+B_{-} \exp \left(-k^{\prime} x\right),(2.2)$

$\Psi_{\mathrm{III}}(x)=C_{+} \exp (i k x)+C_{-} \exp (-i k x) \cdot(2.3)$

Here

$k=2 \pi / \hbar \sqrt{2 m E}, k^{\prime}=2 \pi / \hbar \sqrt{2 m\left(U_{0}-E\right) .}(2.4)$

At the boundary $x=0$ we have the following boundary conditions:

$\Psi_{\mathbf{I}}(0)=\Psi_{\mathrm{II}}(0), \quad \partial \Psi_{\mathbf{I}}(x) /\left.\partial x\right|_{x=0}=\partial \Psi_{\mathrm{II}}(x) /\left.\partial x\right|_{x=0}$

At the boundary $x=l$ we have the following boundary conditions:

$\Psi_{\mathrm{II}}(l)=\Psi_{\mathrm{III}}(l), \quad \partial \Psi_{\mathrm{II}}(x) /\left.\partial x\right|_{x=l}=\partial \Psi_{\mathrm{III}}(x) /\left.\partial x\right|_{x=l} \cdot(2.6)$

From the boundary conditions (2.5)-(2.6) one obtain [8]:

$$
\begin{array}{r}
B_{+}=A / 2\left(1+i k / k^{\prime}\right), B_{-}=A / 2\left(1-i k / k^{\prime}\right), \\
C_{+}=A\left[\cosh \left(k^{\prime} l\right)+i D \sinh \left(k^{\prime} l\right)\right], C_{-}=i A S \sinh \left(k^{\prime} l\right) \exp (i k l),(2.7) \\
D=1 / 2\left(k / k^{\prime}-k^{\prime} / k\right), S=1 / 2\left(k / k^{\prime}+k^{\prime} / k\right) .
\end{array}
$$

From (2.7) one obtain the conservation law: $|A|^{2}=\left|C_{+}\right|^{2}-\left|C_{-}\right|^{2}$.

Let us introduce now a function $E(x, l)$ : 
$E(x, l)=\left(\pi r_{c}^{2}\right)^{-1 / 4} \exp \left(x^{2} / 2 r_{c}^{2}\right)$, for $-\infty<x<l / 2$,

$E(x, l)=\left(\pi r_{c}^{2}\right)^{-1 / 4} \exp \left((x-l)^{2} / 2 r_{c}^{2}\right)$, forl $/ 2 \leq x<+\infty .(2.8)$

Assumption 2.1. We assume now that: (i) at instant $t=0$ the wave function $\Psi_{\mathrm{I}}(x)$ experiences a sudden jump $\quad \Psi_{\mathrm{I}}(x) \rightarrow \Psi_{\mathrm{I}}^{\#}(x)$ of the form

$\Psi_{\mathrm{I}}^{\#}(x)=\mathcal{R}_{\mathrm{I}}(\tilde{x}) \Psi_{\mathrm{I}}(x)\left[\left\|\mathcal{R}_{\mathrm{I}}(\tilde{x}) \Psi_{\mathrm{I}}(x)\right\|_{2}\right]^{-1},(2.9)$

where $\mathcal{R}_{\mathrm{I}}(\tilde{x})$ is a linear operator which is chosen equal to:

$\mathcal{R}_{\mathrm{I}}(\tilde{x})=\left(\pi r_{c}^{2}\right)^{-1 / 4} \exp \left(\hat{x}^{2} / 2 r_{c}^{2}\right),(2.10)$

(ii) at instant $t=0$ the wave function $\Psi_{\mathrm{II}}(x)$ experiences a sudden jump $\quad \Psi_{\mathrm{II}}(x) \rightarrow \Psi_{\mathrm{II}}^{\#}(x)$ of the form

$\Psi_{\mathrm{II}}^{\#}(x)=\mathcal{R}_{\mathrm{II}}(\tilde{x}) \Psi_{\mathrm{II}}(x)\left[\left\|\mathcal{R}_{\mathrm{II}}(\tilde{x}) \Psi_{\mathrm{II}}(x)\right\|_{2}\right]^{-1},(2.11)$

where $\mathcal{R}_{\mathrm{II}}(\tilde{x})$ is a linear operator which is chosen equal to:

$\mathcal{R}_{\mathrm{II}}(\tilde{x})=E(\hat{x}, l),(2.12)$

(iii) at instant $t=0$ the wave function $\Psi_{\mathrm{III}}(x)$ experiences a sudden jump $\quad \Psi_{\mathrm{III}}(x) \rightarrow \Psi_{\mathrm{III}}^{\#}(x)$ of the form

$\Psi_{\mathrm{III}}^{\#}(x)=\mathcal{R}_{\mathrm{III}}(\tilde{x}) \Psi_{\mathrm{III}}(x)\left[\left\|\mathcal{R}_{\mathrm{III}}(\tilde{x}) \Psi_{\mathrm{III}}(x)\right\|_{2}\right]^{-1},(2.13)$

where $\mathcal{R}_{\mathrm{I}}(\tilde{x})$ is a linear operator which is chosen equal to:

$\mathcal{R}_{\text {III }}(\tilde{x})=\left(\pi r_{c}^{2}\right)^{-1 / 4} \exp \left((\hat{x}-l)^{2} / 2 r_{c}^{2}\right) .(2.14)$

Remark 2.1. Note that. We have choose operators (2.10),(2.12) and (2.14) such that the boundary conditions (2.5),(2.6) is satisfied.

\section{Resolution of the Schrödinger'sCat paradox}

Let $\left|s_{1}(t)\right\rangle$ and $\left|s_{2}(t)\right\rangle$ be

$$
\left.\left|s_{1}(t)\right\rangle=\mid \text { undecayed nucleus at instant } t\right\rangle
$$

And

$\left|s_{2}(t)\right\rangle=\mid$ decayed nucleus at instant $\left.t\right\rangle(3.1)$

correspondingly. In a good approximation we assume now that 
$\left|s_{1}(0)\right\rangle=\Psi_{\mathrm{II}}^{\#}(x)(3.2)$

and

$\left|s_{2}(0)\right\rangle=\Psi_{\mathbf{I}}^{\#}(x) \cdot(3.3)$

Remark 3.1. Note that: (i) $\left|s_{2}(0)\right\rangle=\mid$ decayed nucleus at instant 0$\rangle==\mid$ free $\alpha-$ particle at instant 0$\rangle$. (ii) Feynman propagator of a free $\alpha$-particle inside regionI are [9]:

$K_{\mathbf{I}}\left(x, t, x_{0}\right)=\left(\frac{m}{2 \pi i \hbar t}\right)^{1 / 2} \exp \left\{\frac{i}{\hbar}\left[\frac{m\left(x-x_{0}\right)^{2}}{2 t}\right]\right\} \cdot(3 \cdot 4)$

Therefore from Eq.(3.3),Eq.(2.9) and Eq.(3.4) we obtain

$$
\begin{array}{r}
\left|s_{2}(t)\right\rangle=\Psi_{I}^{\#}(x, t)=\int_{-\infty}^{0} \Psi_{\mathbf{I}}^{\#}(x) K_{\mathbf{I}}\left(x, t, x_{0}\right) d x_{0}= \\
=\left(\pi r_{c}^{2}\right)^{-1 / 4}\left(\frac{m}{2 \pi i \hbar t}\right)^{1 / 2} \int_{-\infty}^{0} d x_{0} \exp \left(-\frac{x_{0}^{2}}{2 r_{c}^{2}}\right) \exp \left\{\frac{i}{\hbar}\left[S\left(x, t, x_{0}\right)\right]\right\} \cdot(3.5)
\end{array}
$$

Here

$S\left(x, t, x_{0}\right)=\frac{m\left(x-x_{0}\right)^{2}}{2 t}-\pi \sqrt{8 m E} x_{0} \cdot(3.6)$

We assume now that

$\hbar \ll 2 r_{c}^{2} \ll 1 .(3.7)$

Oscillatory integral in RHS of Eq.(3.5) is calculated now directly using stationary phaseapproximation.

The phase term $S\left(x, t, x_{0}\right)$ given by Eq.(3.6) is stationary when

$\frac{\partial S\left(x, t, x_{0}\right)}{\partial x_{0}}=-\frac{m\left(x-x_{0}\right)}{2 t}-\pi \sqrt{8 m E}=0$.

Therefore

$-\left(x-x_{0}\right)=\pi t \sqrt{8 E / m}(3.9)$

and thus stationary point $x_{0}(t, x)$ are

$x_{0}(t, x)=\pi t \sqrt{8 E / m}+x .(3.10)$

Thus from Eq.(3.5) and Eq.(3.10) using stationary phase approximation we obtain

$\left|s_{2}(t)\right\rangle=\left(\pi r_{c}^{2}\right)^{-1 / 4} \exp \left(-\frac{x_{0}^{2}(t, x)}{2 r_{c}^{2}}\right) \exp \left\{\frac{\mathrm{i}}{\hbar}\left[S\left(\mathrm{x}, \mathrm{t}, \mathrm{x}_{0}(t, x)\right)\right]\right\}+O(\hbar) .(3$

Here 
$S\left(\mathrm{x}, \mathrm{t}, \mathrm{x}_{0}(t, x)\right)=\frac{m\left(x-x_{0}(t, x)\right)^{2}}{2 t}-\pi \sqrt{8 m E} x_{0}(t, x)$

FromEq.(3.10)-Eq.(3.11) we obtain

$$
\begin{aligned}
& \left.\qquad s_{2}(t)|\hat{x}| s_{2}(t)\right\rangle \approx\left(\pi r_{c}^{2}\right)^{-1 / 2} \int_{-\infty}^{+\infty} d x x \exp \left[-\frac{(\pi t \sqrt{8 E / m}+x)}{2 r_{c}^{2}}\right]= \\
& =-\pi t \sqrt{\frac{8 E}{m}} . \text { (3.13) }
\end{aligned}
$$

Remark 3.2. From Eq.(3.13) follows directly that $\alpha$-particle at eachinstant $t \geq 0$ moves quasi-classically from right to left by the law

$x(t)=-\pi t \sqrt{\frac{8 E}{m}},(3.14)$

i.e.estimating the position $x(t)$ at each instant $t \geq 0$ with final error $r_{c}$ gives $|\langle x(t)\rangle-x(t)| \leq r_{c}$, with a probabilityP $\left\{|\langle x(t)\rangle-x(t)| \leq r_{c}\right\} \cong 1$.

Remark 3.3. We assume now that a distance between radioactive source andinternal monitor which detects

a single atom decaying (see Pic.1.1) is equal to $L$.

Proposition 3.1. After $\alpha$-decay the collapse:|live cat $\rangle \rightarrow \mid$ death cat $\rangle$ arises atinstant $T_{\text {col }}$.

$T_{\text {col. }} \cong \frac{L}{\pi \sqrt{8 m E}} \cdot(3.15)$

with a probability $P_{\boldsymbol{T}_{\text {col. }}}\{\mid$ death cat $\left.\rangle\right\}$ to observe a state $\mid$ death cat $\rangle$ at instant $T_{\text {col. }}$ is $P_{T_{\text {col. }}}\{\mid$ death cat $\left.\rangle\right\} \cong 1$.

Suppose now that a nucleus $\boldsymbol{n}$ whose Hilbert space is spanned by orthonormal states $\left|s_{i}(t)\right\rangle, i=1,2$

where $\left|s_{1}(t)\right\rangle=\mid$ undecayed nucleus at instant $\left.t\right\rangle$ and $\left|s_{2}(t)\right\rangle=\mid$ decayed nucleus at instant $\left.t\right\rangle$ is in the superposition state,

$\left|\Psi_{t}\right\rangle_{n}=c_{1}\left|s_{1}(t)\right\rangle+c_{2}\left|s_{2}(t)\right\rangle,\left|c_{1}\right|^{2}+\left|c_{2}\right|^{2}=1 .(3.16)$

Remark 3.4. Note that: (i) $\left|s_{2}(0)\right\rangle=\mid$ uncayed nucleus at instant 0$\rangle=$ $=\mid \alpha-$ particle at instant 0 inside region II $\rangle$. (ii) Feynman propagator $\alpha$-particle inside region II are [9]:

$K_{\mathbf{I I}}\left(x, t, x_{0}\right)=\left(\frac{m}{2 \pi i \hbar t}\right)^{1 / 2} \exp \left\{\frac{i}{\hbar} S\left(x, t, x_{0}\right)\right\}$. 
Here

$S\left(x, t, x_{0}\right)=\frac{m\left(x-x_{0}\right)^{2}}{2 t}+m t\left(U_{0}-E\right) \cdot(3.18)$

Therefore from Eq.(2.11)-Eq.(2.12), and Eq.(3.2) and Eq.(3.17) we obtain

$$
\begin{array}{r}
\left|s_{1}(t)\right\rangle=\Psi_{I I}^{\#}(x, t)=\int_{0}^{l} \Psi_{\text {II }}^{\#}(x) K_{\mathbf{I I}}\left(x, t, x_{0}\right) d x_{0}= \\
=\left(\pi r_{c}^{2}\right)^{-1 / 4}\left(\frac{m}{2 \pi i \hbar t}\right)^{1 / 2} \int_{0}^{l} d x_{0} E\left(x_{0}, l\right) \theta_{l}\left(x_{0}\right) \Psi_{\text {II }}^{\#}(x) \exp \left\{\frac{i}{\hbar} S\left(x, t, x_{0}\right)\right\},(3.1
\end{array}
$$

where

$$
\theta_{l}\left(x_{0}\right)=\left\{\begin{array}{l}
1 \text { for } x \in[0, l] \\
0 \text { for } x \notin[0, l]
\end{array}\right.
$$

Remark 3.5. We assume for simplification now that

$l \hbar^{-1} \leq 1 .(3.20)$

Thus oscillatory integral in RHS of Eq.(3.19) is calculated now directly usingstationary phase approximation. The phase term $S\left(x, t, x_{0}\right)$ given by Eq.(3.18) isstationary when

$\frac{\partial S\left(x, t, x_{0}\right)}{\partial x_{0}}=-\frac{m\left(x-x_{0}\right)}{2 t}=0 .(3.21)$

and therefore stationary point $x_{0}(t, x)$ are

$x_{0}(t, x)=x \cdot(3.22)$

Therefore from Eq.(3.19) and Eq.(3.22) using stationary phase approximation weobtain

$\left|s_{1}(t)\right\rangle=\Psi_{\mathrm{II}}^{\#}(x, t)=O(1) E(x, l) \theta_{l}(x) \exp \left\{\frac{i}{\hbar} m t\left(U_{0}-E\right)\right\}+O(\hbar) .(3.23)$

Therefore fromEq.(3.23) and (3.20) we obtain

$\left\langle s_{1}(t)|\hat{x}| s_{1}(t)\right\rangle=O(1) E(x, l) \theta_{l}(x)+O(\hbar)=O(\hbar) .(3.24)$

Proposition3.2. Suppose that a nucleus $\mathrm{n}$ is in the superposition state given byEq.(3.16). Then the collapse:|live cat $\rangle \rightarrow \mid$ death cat $\rangle$ arises at instant $T_{\text {col }}$.

$T_{\text {col. }} \cong \frac{L}{\pi\left|c_{2}\right|^{2} \sqrt{8 m E}}(3.25)$

with a probability $P_{T_{\text {col. }}}\{\mid$ death cat $\left.\rangle\right\}$ to observe a state |death cat $\rangle$ at instant $T_{\text {col. }}$ is $P_{T_{\text {col. }}}\{\mid$ death cat $\left.\rangle\right\} \cong 1$. 
Proof. From Eq.(3.16), Eq.(3.11),Eq.(3.13),Eq.(3.23)-Eq.(3.24) weobtain

$$
\begin{gathered}
{ }_{n}\left\langle\Psi_{\mathrm{t}} \mid \Psi_{\mathrm{t}}\right\rangle_{\boldsymbol{n}}=\left|c_{1}\right|^{2}\left\langle s_{1}(t)|\hat{x}| s_{1}(t)\right\rangle+\left|c_{2}\right|^{2}\left\langle s_{2}(t)|\hat{x}| s_{2}(t)\right\rangle+ \\
+c_{1} c_{2}^{*}\left\langle s_{2}(t)|\hat{x}| s_{1}(t)\right\rangle+c_{2} c_{1}^{*}\left\langle s_{1}(t)|\hat{x}| s_{2}(t)\right\rangle=\left|c_{2}\right|^{2}\left\langle s_{2}(t)|\hat{x}| s_{2}(t)\right\rangle+O(\hbar) .(3.26)
\end{gathered}
$$

From Eq.(3.26) one obtain

$$
\left\langle T_{\text {col. }}\right\rangle \cong \frac{L}{\pi\left|c_{2}\right|^{2} \sqrt{8 m E}} \cdot(3.27)
$$

Let us consider now a state $\left|\Psi_{t}\right\rangle_{n}$ given by Eq.(3.16). This state consists of asum two Gaussian wave packets: $c_{1} \Psi_{\mathrm{II}}^{\#}(x, t)$ and $c_{2} \Psi_{\mathrm{I}}^{\#}(x, t)$. Wave packet $c_{2} \Psi_{\mathrm{II}}^{\#}(x, t)$ present an $\alpha_{\mathrm{II}}$-particle which live inside regionII see Pic.2.1. Wave packet $c_{2} \Psi_{\mathrm{I}}^{\#}(x, t)$ present an $\alpha_{\mathrm{I}}$-particle which moves inside region I from the right to left, see Pic.2.1.Note that In II = $\emptyset$.From Eq.(B.5) (see Appendix B) we obtain that: the probability $P(x, d x, t)$ of the $\alpha_{\mathbf{I}}$-particlebeing observed to have a coordinate in the range $x$ to $x+d x$ at instant $t$ is

$P(x, t)=\left|c_{2}\right|^{-2} \Psi_{\mathbf{I}}^{\#}\left(x\left|c_{2}\right|^{-2}, t\right) d x \cdot(3.28)$

From Eq.(3.28) and Eq. (3.11) follows that $\alpha_{\mathrm{I}}$-particle at each instant $t \geq 0$ moves quasi-classically from right to left by the law

$x(t)=-\pi t\left|c_{2}\right|^{2} \sqrt{\frac{8 E}{m}}(3.29)$

at the uniform velocity $\pi\left|c_{2}\right|^{2} \sqrt{\frac{8 E}{m}}$.Equality (3.29) completed the proof.

Remark 3.6. We remain now that: there are widespread claims that Schrödinger'scat is not in a definite alive or dead state but is, instead, in a superposition of the two[6],[7],[10]:

$\mid$ cat $\rangle=c_{1} \mid$ live cat $\rangle+c_{2} \mid$ death cat $\rangle$.

Proposition 3.3. (i) Assume now that: a nucleus $\mathbf{n}$ is in the superposition state isgiven by Eq.(3.16) and Schrödinger's cat is in a state|live cat $\rangle$. Then collapse |live cat $\rangle \rightarrow \mid$ death cat $\rangle$ arises at instant $t=T_{\text {col. }}$ is given by Eq.(3.25).(ii) Assume now that: a nucleus $\mathbf{n}$ is in the superposition state is given by Eq.(3.16) and Schrödinger's cat is, instead, in a superposition of the two:

$\mid$ cat at instant $t\rangle=c_{1} \mid$ live cat atinstant $\left.t\right\rangle+c_{2} \mid$ death cat at instant $\left.t\right\rangle$.

Then collapse|live cat $\rangle \rightarrow \mid$ death cat $\rangle$ arises at instant $t=T_{\text {col. }}$ is given by Eq.(3.25).

Proof. (i) Immediately follows from Proposition 3.2. (ii) Immediately follows from statement (i).

Thus actually is the collapsed state of both the Schrödinger's cat and thenucleus at each instant $t \geq$ $T_{c o l}$. always shows definite and predictableoutcomes even if cat also consists of a superposition: $\mid$ cat $\rangle=c_{1} \mid$ live cat $\rangle+c_{2} \mid$ death cat $\rangle$. Contrary to van Kampen's [10] and some others' opinions, 
"looking" at the outcomechanges nothing, beyond informing the observer of what has already happened.

\section{Conclusions}

The canonical formulation of the cat state:

$$
\left.\left.\left.\left.\mid \text { cat }\rangle=c_{1} \mid \text { live cat }\right\rangle \mid \text { undecayed nucleus }\right\rangle+c_{2} \mid \text { death cat }\right\rangle \mid \text { decayed nucleus }\right\rangle
$$

completely obscures the unitary Schrödinger evolution which by using GRW collapse model, predicts specific nonlocal entanglement [7]. The cat state must be written as:

$$
\begin{gathered}
\left.\left.\mid \text { cat at instant } t\rangle=c_{1} \mid \text { live cat at instant } t\right\rangle \mid \text { undecayed nucleus at instant } t\right\rangle+ \\
\left.\left.+c_{2} \mid \text { death cat at instant } t\right\rangle \mid \text { decayed nucleus at instant } t\right\rangle
\end{gathered}
$$

This entangled state actually is the collapsed state of both the cat and the nucleus, showing definite outcomes at each instant $t \geq T_{\text {col }}$.

\section{Acknowledgments}

A reviewer provided important clarification.

\section{Appendix A}

Suppose we have an observable $Q$ of a system that is found, for instance through an exhaustive series of measurements, to have a continuous range of values $\theta_{1} \leq q \leq \theta_{2}$. Then we claim the following postulates:

Postulate1. Any given quantum system is identified with some infinite-dimensional Hilbert space H.

Definition 1.The pure states correspond to vectors of norm 1. Thus the set of all pure states corresponds to the unit sphere $\mathbf{S}^{\infty} \subset \mathbf{H}$ in the Hilbert space $\mathbf{H}$.

Definition 2.The projective Hilbert space $P(\mathbf{H})$ of a complex Hilbert space $\mathbf{H}$ is the set of equivalence classes $[\mathbf{v}]$ of vectors $\mathbf{v}$ in $\mathrm{H}$, with $\mathbf{v} \neq 0$, for the equivalence relation given by $\mathbf{v} \sim_{P} \mathbf{w} \Leftrightarrow \mathbf{v}=\lambda \mathbf{w}$ for some non-zero complex number $\lambda \in \mathbb{C}$. The equivalence classes for the relation $\sim_{P}$ are also called rays or projective rays.

Remark 1.The physical significance of the projective Hilbert space $P(\mathbf{H})$ is that in canonical quantum theory, the states $|\psi\rangle$ and $\lambda|\psi\rangle$ represent the same physical state of the quantum system, for any $\lambda \neq 0$.

It is conventional to choose a state $|\psi\rangle$ from the ray $[|\psi\rangle]$ so that it has unit norm $\langle\psi \mid \psi\rangle=1$.

Remark 2. In contrast with canonical quantum theory we have used also contrary to $\sim_{P}$ equivalence relation $\sim_{Q}$, see Def.A.3. 
Postulate 2.The states $\left\{|q\rangle \mid \theta_{1} \leq q \leq \theta_{2}\right\}$ form a complete set of $\delta$-function normalized basis states for the state space $\mathbf{H}$ of the system.

Remark 3.The states $\left\{|q\rangle \mid \theta_{1} \leq q \leq \theta_{2}\right\}$ form a complete set of basis states means that any state $|\psi\rangle \in$ Hof the system can be expressed as $|\psi\rangle=\int_{\theta_{1}}^{\theta_{2}} c_{\psi}(q)|q\rangle d q$ while $\delta$-function normalized means that $\left\langle q \mid q^{\prime}\right\rangle=\delta\left(q-q^{\prime}\right)$ from which follows $c_{\psi}(q)=\langle q \mid \psi\rangle_{\text {so that }}$

$|\psi\rangle=\int_{\theta_{1}}^{\theta_{2}}|q\rangle\langle q \mid \psi\rangle d q$.The completeness condition can then be written as $\int_{\theta_{1}}^{\theta_{2}}|q\rangle\langle q| d q=\hat{1}$.

Completeness means that for any state $|\psi\rangle \in \mathbf{S}^{\infty}$ it must be the case that $\left.\int_{\theta_{1}}^{\theta_{2}}|q| \psi\right|^{2} d q \neq 0$.

Postulate3.For the system in a pure state $|\psi\rangle \in \mathbf{S}^{\infty}$ the probability $P(q, d q,|\psi\rangle)$ of obtainingthe result $q$ lying in the range $(q, q+d q)$ on measuring $Q$ is

$P(q, d q,|\psi\rangle)=\left|c_{\psi}(q)\right|^{2} d q .($ A.1 $)$

Postulate 4.The observable $Q$ is represented by a Hermitean operator $\hat{Q}$ whose eigenvalues are thepossible results $\left\{q \mid \theta_{1} \leq q \leq \theta_{2}\right\}$,of a measurement of $Q$, and the associated eigenstates arethe states $\left\{|q\rangle \mid \theta_{1} \leq q \leq \theta_{2}\right\}$, i.e. $\hat{Q}|q\rangle=q|q\rangle$.

Remark 4. The spectral decomposition of the observable $\hat{Q}$ is then $\hat{Q}=\int_{\theta_{1}}^{\theta_{2}} q|q\rangle\langle q| d q$. (A.2)

Postulate 5.(von Neumann measurement postulate) Assume that $|\psi\rangle \in \mathbf{S}^{\infty}$. Then if onperforming a measurement of $Q$ with an accuracy $\delta q$, the result is obtained in therange $\left(q-\frac{1}{2} \delta q, q+\frac{1}{2} \delta q\right)$,then the system will end up in the state

$\frac{\hat{P}(q, \delta q)|\psi\rangle}{\sqrt{\langle\psi|\hat{P}(q, \delta q)| \psi\rangle}}(\mathrm{A} .3)$

where $\hat{P}(q, \delta q)=\int_{q-\frac{1}{2} \delta q}^{q+\frac{1}{2} \delta q}\left|q^{\prime}\right\rangle\left\langle q^{\prime}\right| d q^{\prime}$

Postulate 6. For the system in state $\left|\psi^{a}\right\rangle=a|\psi\rangle$, where $|\psi\rangle \in \mathbf{S}^{\infty}, a \in \mathbb{C}, a \neq 1$ and $|\psi\rangle=$ $\int_{\theta_{1}}^{\theta_{2}} c_{\psi}(q)|q\rangle d q$ the probability $P\left(q, d q,\left|\psi^{a}\right\rangle\right)$ of obtaining the result $q$ lying in the range $(q, q+d q)$ on measuring $Q$ is

$P\left(q, d q,\left|\psi^{a}\right\rangle\right)=|a|^{-2}\left|c_{\psi}\left(q|a|^{-2}\right)\right|^{2} d q$. (A.3)

Remark A.3. Formal motivation of the Postulate6 is a very simple and clear. Let $\left|\psi_{t}^{\boldsymbol{a}}\right\rangle, t \in[0, \infty)$ be a state 
$\left|\psi_{t}^{a}\right\rangle=a\left|\psi_{t}\right\rangle$,where $\left|\psi_{t}\right\rangle \in \mathbf{S}^{\infty}, a \in \mathbb{C}, a \neq 1$ and $\left|\psi_{t}\right\rangle=\int_{\theta_{1}}^{\theta_{2}} c_{\psi_{t}}(q)|q\rangle d q=\int_{\theta_{1}}^{\theta_{2}} c(q, t)|q\rangle d q$

\section{Note that:}

(i) any result of the process of continuous measurements on measuring $Q$ for the system instate $\left|\psi_{t}\right\rangle$

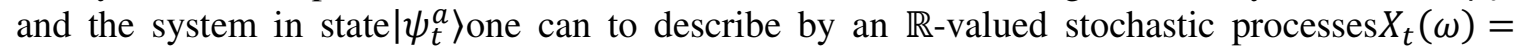
$X_{t}\left(\omega,\left|\psi_{t}\right\rangle\right)$ and

$Y_{t}^{a}(\omega)=Y_{t}^{a}\left(\omega,\left|\psi_{t}^{a}\right\rangle\right)$ such that both processes is given on an probability $\operatorname{space}(\Omega, \mathcal{F}, \mathbf{P})$ and a measurable

$\operatorname{space}(\mathbb{R}, \Sigma)$

(ii) We assume now that $\forall \Theta \in \mathcal{F}$ :

$\mathbf{E}_{\Theta}\left[X_{t}(\omega)\right]=\int_{\Theta} X_{t}(\omega) d \mathbf{P}(\omega)=\mathbf{E}_{\Theta}\left[X_{t}\left(\omega,\left|\psi_{t}\right\rangle\right)\right]=\left\langle\psi_{t}\left|\hat{Q}_{\Delta(\Theta)}\right| \psi_{t}\right\rangle,(\mathrm{A} .4)$

$\mathbf{E}_{\Theta}\left[Y_{t}^{a}(\omega)\right]=\int_{\Theta} Y_{t}^{a}(\omega) d \mathbf{P}(\omega)=\mathbf{E}_{\Theta}\left[Y_{t}^{a}\left(\omega,\left|\psi_{t}^{a}\right\rangle\right)\right]=\left\langle\psi_{t}^{a}\left|\hat{Q}_{\Delta(\Theta)}\right| \psi_{t}^{a}\right\rangle=$ $|a|^{2}\left\langle\psi_{t}\left|\hat{Q}_{\Delta(\Theta)}\right| \psi_{t}\right\rangle,(\mathrm{A} .5)$

where $\Delta: \mathcal{F} \leadsto \Sigma$ is a $\sigma$-gomomorfizmsuch that $\Delta(\mathcal{F}) \subseteq \Sigma$ and $\widehat{Q}_{\Delta(\Theta)}=\int_{\Delta(\Theta)} q|q\rangle\langle q| d q$.

(iii) From Eq.(A.4)- Eq.(A.5),by using Radon-Nikodym theorem, one obtain

$Y_{t}^{a}(\omega)=|a|^{2} X_{t}(\omega)$ (A.6)

(iv) Let $\rho_{t}(x)$ be a probability density of the stochastic process $X_{t}(\omega)$ and let $\rho_{t}^{a}(y)$

be a probability density of the stochastic process $Y_{t}^{a}(\omega)$.From Eq.(A.6) one obtain directly

$\rho_{t}^{a}(y)=|a|^{-2} \rho_{t}\left(y|a|^{-2}\right) .($ A.7)

Definition 3.Let $\left|\psi^{a}\right\rangle$ be a state $\left|\psi^{a}\right\rangle=a|\psi\rangle$, where $|\psi\rangle \in \mathbf{S}^{\infty}, a \in \mathbb{C}, a \neq 1$ and $|\psi\rangle=$ $\int_{\theta_{1}}^{\theta_{2}} c_{\psi}(q)|q\rangle d q$ and let $\left|\psi_{a}\right\rangle$ be an statesuch that $\left|\psi_{a}\right\rangle \in \mathbf{S}^{\infty}$. States $\left|\psi^{a}\right\rangle$ and $\left|\psi_{a}\right\rangle$ is a $Q$-equivalent: $\left|\psi_{a}\right\rangle \sim_{Q}\left|\psi^{a}\right\rangle$

iff $\forall q \in\left[\theta_{1}, \theta_{2}\right]$

$P\left(q, d q,\left|\psi^{a}\right\rangle\right)=|a|^{-2}\left|c_{\psi_{a}}\left(q|a|^{-2}\right)\right|^{2} d q$

Postulate 7.For any state $\left|\psi^{a}\right\rangle=a|\psi\rangle$, where $|\psi\rangle \in \mathbf{S}^{\infty}, a \in \mathbb{C}, a \neq 1$ and $|\psi\rangle=\int_{\theta_{1}}^{\theta_{2}} c_{\psi}(q)|q\rangle d q$ thereexist an state $\left|\psi_{a}\right\rangle \in \mathbf{S}^{\infty}$ such that $\left|\psi_{a}\right\rangle \sim_{Q}\left|\psi^{a}\right\rangle$.

\section{AppendixB.Position observable of a particle inone dimension}

The position representation is used in quantum mechanical problems where it isthe position of the 
particle in space that is of primary interest. For this reason, theposition representation, or the wave function, is the preferred choice of representation.

B.1. In one dimension, the position $x$ of a particle can range over the values $-\infty<x<\infty$ Thus the Hermitean operator $\hat{x}$ corresponding to this observable willhave eigenstates $|x\rangle$ and associated eigenvalues $x$ such that: $\hat{x}|x\rangle=x|x\rangle$.

B.2. As the eigenvalues cover a continuous range of values, the completenessrelation will be expressed

as an integral:

$\left|\psi_{t}\right\rangle=\int_{-\infty}^{\infty}|x\rangle\left\langle x \mid \psi_{t}\right\rangle d x .($ B. 1$)$

Here $\left\langle x \mid \psi_{t}\right\rangle=\psi(x, t)$ isthe wave function associated with the particle at each instant $t$. Since there is acontinuously infinite number of basis states $|x\rangle$,these states are $\delta$-function normalized

$$
\left\langle x \mid x^{\prime}\right\rangle=\delta\left(x-x^{\prime}\right) \text {. }
$$

B.3. The operator $\hat{x}$ itself can be expressed as

$\hat{x}=\int_{-\infty}^{\infty} x|x\rangle\langle x| d x .($ B.2)

B.4. The wave function is, of course, just the components of the state vector $\left|\psi_{t}\right\rangle \in \mathbf{S}^{\infty}$, with respect to the position eigenstates as basis vectors. Hence, the wavefunction is often referred to as being the state of the system in the positionrepresentation. The probability amplitude $\left\langle x \mid \psi_{t}\right\rangle$ is just the wave function, written $\psi(x, t)$ and is such that $|\psi(x, t)|^{2} d x$ is the probability $P\left(x, d x, t ;\left|\psi_{t}\right\rangle\right)$ of the particlebeing observed to have a coordinate in the range $x$ to $x+d x$.

Definition B.1.Let $\left|\psi_{\boldsymbol{t}}^{\boldsymbol{a}}\right\rangle, t \in[0, \infty)$ be a state $\left|\psi_{t}^{a}\right\rangle=a\left|\psi_{t}\right\rangle$, where $\left|\psi_{t}\right\rangle \in \mathbf{S}^{\infty}, a \in \mathbb{C}, a \neq 1$ and $\left|\psi_{t}\right\rangle=\int_{-\infty}^{\infty} \psi(x, t)|x\rangle d x$. Let $\left|\psi_{t, a}\right\rangle$ be an state such that $\left.\forall t \in \llbracket 0, \infty\right):\left|\psi_{t, a}\right\rangle \in \mathbf{S}^{\infty}$.States $\left|\psi_{\boldsymbol{t}}^{\boldsymbol{a}}\right\rangle$ and $\left|\psi_{t, a}\right\rangle$ is called $x$-equivalent: $\left|\psi_{\boldsymbol{t}}^{\boldsymbol{a}}\right\rangle \sim_{x}\left|\psi_{t, a}\right\rangle$ iff

$P\left(x, d x, t ;\left|\psi_{t}^{a}\right\rangle\right)=|a|^{-2}\left|\psi\left(x|a|^{-2}, t\right)\right|^{2} d x=P\left(x, d x, t ;\left|\psi_{t, a}\right\rangle\right) .(\mathrm{B} .3)$

B.5.From Postulate A.7 (see Appendix A) follows that: for any state $\left|\psi_{t}^{a}\right\rangle=a\left|\psi_{t}\right\rangle$, where $\left|\psi_{t}\right\rangle \in$ $\mathbf{S}^{\infty}, a \in \mathbb{C}, a \neq 1$ and $\left|\psi_{t}\right\rangle=\int_{-\infty}^{\infty} \psi(x, t)|x\rangle d x$ there exist an state $\left|\psi_{t, a}\right\rangle \in \mathbf{S}^{\infty}$ such that

$$
\left|\psi_{t}^{\boldsymbol{a}}\right\rangle \sim_{x}\left|\psi_{t, a}\right\rangle
$$

Definition B.2.A pure state $\left|\psi_{t}\right\rangle \in \mathbf{S}^{\infty}$, where $\left|\psi_{t}\right\rangle=\int_{-\infty}^{\infty} \psi(x, t)|x\rangle d x$ is called a weakly Gaussian in the position representation iff

$|\psi(x, t)|^{2}=\frac{1}{\sigma_{t} \sqrt{2 \pi}} \exp \left[-\frac{\left(x-\left\langle x_{t}\right\rangle\right)^{2}}{\sigma_{t}^{2}}\right],(\mathrm{B} .4)$

where $\left\langle x_{t}\right\rangle$ and $\sigma_{t}$ an functions which depend only on variable $t$. 
B.6. From Postulate A.7 (see Appendix A) follows that: for any state $\left|\psi_{t}^{a}\right\rangle=a\left|\psi_{t}\right\rangle$, where $\left|\psi_{t}\right\rangle \in \mathbf{S}^{\infty}, a \in \mathbb{C}, a \neq 1$ and $\left|\psi_{t}\right\rangle=\int_{-\infty}^{\infty} \psi(x, t)|x\rangle d x$ is a weakly Gaussian state in the position

Representation, the probability $P\left(x, d x, t ;\left|\psi_{t}^{a}\right\rangle\right)$ of the particle being observed to have a coordinate

in the range $x$ to $x+d x$ is

$P\left(x, d x, t ;\left|\psi_{t}^{a}\right\rangle\right)=\frac{1}{|a|^{2} \sigma_{t} \sqrt{2 \pi}} \exp \left[-\frac{\left(x|a|^{-2}-\left\langle x_{t}\right\rangle\right)^{2}}{\sigma_{t}^{2}}\right] .(\mathrm{B} .5)$

B.7. From Postulate A.7 (see Appendix A) follows that: for any state $\left|\psi_{t}^{a}\right\rangle=a\left|\psi_{t}\right\rangle$, where $\left|\psi_{t}\right\rangle \in \mathbf{S}^{\infty}, a \in \mathbb{C}, a \neq 1$ and $\left|\psi_{t}\right\rangle=\int_{-\infty}^{\infty} \psi(x, t)|x\rangle d x$ is a weakly Gaussian state in the position

Representation, there exist a weakly Gaussian state $\left|\psi_{t, a}\right\rangle \in \mathbf{S}^{\infty}$ such that

$P\left(x, d x, t ;\left|\psi_{t}^{a}\right\rangle\right)=P\left(x, d x, t ;\left|\psi_{t, a}\right\rangle\right)$.(B.6)

\section{References}

[1] S. Weinberg, Phys. Rev. A 85, 062116 (2012).

[2] A. Bassi, K. Lochan, S. Satin, T. P. Singh, H. Ulbricht, Models of wave function Collapse,Underlying Theories, and Experimental Tests,Rev. Mod. Phys. 85, $471-527$ (2013) http://arxiv.org/abs/1204.4325

[3] K.Jacobs, D. A. Steck, A Straightforward Introduction to Continuous Quantum Measurement, Contemporary Physics 47, 279 (2006) DOI:10.1080/00107510601101934

[4] M.B.Mensky,Continuous Quantum Measurements and Path Integrals,Hardcover: 188 pp. Publisher: CRC Press (January 1, 1993) ISBN-10: 0750302283 ISBN-13: 978-0750302289

[5] M.B.Mensky,Quantum Measurements and Decoherence: Models and Phenomenology,Series: Fundamental Theories of Physics, Vol. 1102000, XVI, 231 pp. ISBN 978-94-015-9566-7

[6] A.J. Leggett, Schrödinger's Cat and Her Laboratory Cousins, Contemp.Phys.,1984, v.25, No.6, pp. 583-598.

[7] A. Hobson, Two-photon interferometry and quantum state collapse,Phys. Rev. A 88, 022105 Published 8 August 2013

[8] G. Gamow,"ZurQuantentheorie des Atomkernes",Z. Physik 51, 204 (1928)

[9] R. Feinman, A. Hibbs, (2005), Quantum Mechanics and Path Integrals, Dover(Emended Edition).

[10] N.G. van Kampen, PhysicaA 153, 97 (1988). 